MATEC Web of Conferences 4, 02001 (2013)

DOI: $10.1051 /$ matecconf/20130402001

(C) Owned by the authors, published by EDP Sciences, 2013

\title{
Resolving the effects of albumin glycation using the quartz crystal microbalance
}

\author{
G.T. Heller ${ }^{1}$, T.J. Zwang ${ }^{1}$, M.H. Sazinsky ${ }^{1}$ and M.S. Johal ${ }^{1 *}$ \\ ${ }^{1}$ Pomona College, Chemistry Department, 645 N. College Avenue, Claremont, CA 91711, USA \\ *malkiat.johal@pomona.edu
}

\begin{abstract}
Both lysine and arginine residues are particularly important at receptor sites for binding anionic ligands. These receptor sites may become compromised via non-enzymatic glycation. While lysine residues are glycated in the presence of glucose, arginine residues are predominantly glycated by $\alpha$-oxoaldehydes like glyoxal. This study used a quartz crystal microbalance with dissipation monitoring (QCM-D) to examine the binding affinity of surface immobilized human serum albumin (HSA) to hemin after the HSA was preincubated with glucose or glyoxal. We found it necessary to pre-expose the HSA functionalized crystal surface to hemin to block irreversible unintended interactions. Glycation with glucose showed little affect on HSA's affinity for hemin, however, modification with glyoxal showed diminished hemin binding capacity. Despite the hemin-blocking step, we were unable to obtain $\mathrm{K}_{\mathrm{d}}$ values consistent with those in literature, which we attribute to other unaccounted for nonspecific interactions. This study highlights the need for a kinetic QCM-D analysis method that accounts for unintended interactions at the sensor surface so that the hemin-blocking step may be eliminated.
\end{abstract}

\section{Introduction}

Several investigations have reported a link between poor glycemic control in diabetics and increased serum iron levels, suggesting a correlation between non-enzymatic glycation and increased serum iron [1-4]. Many studies oversimplify conditions representing poor glycemic control and make unilateral claims about glycation and diabetes based on the effect of an overabundance of glucose on the body. Despite the differences in structure and behavior between glucose and other reactive species, these experiments often fail to consider glycation complications caused by reactive compounds other than glucose [5]. While lysine residues are glycated on human serum albumin (HSA) in the presence of glucose [6], arginine residues are predominantly glycated in the presence of $\alpha$-oxoaldehydes like glyoxal [7], a biproduct of sugar autoxidation that is more prevalent in diabetics than the general population. We hypothesize that due to the difference in glycation tendencies between glyoxal and glucose, they will have different effects on serum iron levels.

Arginine and lysine residues are both commonly found at anionic ligand binding pockets of proteins. Modifying these residues through glycation is potentially damaging because it can diminish the affinity for anionic ligands. One important example of an anionic ligand binding pocket is the heme binding pocket of HSA. Experiments on the effects of glycation with glucose have shown minimal effects on HSA's ability to bind heme [8]. However, due to the prevalence of arginine residues at this location, it is likely that glycation with glyoxal or other sugars could result in a decrease in affinity of HSA for hemin, an iron containing porphyrin. It is well known that iron is a growth-limiting micronutrient for bacteria and that part of the body's innate defense is to sequester as much iron as possible to prevent infection. HSA binds hemin to accomplish this, effectively preventing bacteria from easily accessing iron in free hemin. We hypothesize that the glycation of HSA by sugars known to glycate at arginine residues disrupts HSA's ability to function in iron homeostasis, which would promote bacterial infection by increasing the availability of serum iron.

This study used a quartz crystal microbalance with dissipation monitoring (QCM-D) to examine the binding affinity of hemin to native, glucosylated or glyoxalated HSA. Although it was expected that the interaction between hemin and the HSA functionalized surface would be entirely reversible due to the fact that HSA has an affinity for hemin of approximately $10 \mathrm{nM}$ [9], both reversible and irreversible binding of hemin to the surface were observed, despite extensive rinsing. However, if, after the rinse, the surfaces were re-exposed to hemin, the second exposures display proportionally more reversible binding than the first. Thus, we found it necessary to 
expose hemin to our surfaces twice in order to minimize unintended irreversible binding events.

\section{Materials and methods}

\subsection{Glycation of albumin}

HSA, glucose and glyoxal were obtained from SigmaAldrich and used as received. HSA (2 $\mathrm{mg} / \mathrm{mL})$ was incubated at $37^{\circ} \mathrm{C}$ with $10 \mathrm{mM}$ glucose, $0.7 \mathrm{mM}$ glyoxal or with no sugar for two weeks in phosphate buffered saline (PBS) at $\mathrm{pH} 7.4$.

\subsection{QCM-D}

Real-time frequency and dissipation data were collected using QCM-D (E4, Q-Sense, Gothenburg, Sweden). Frequency data were obtained at the third harmonic. The QCM-D sensor consisted of an AT-cut piezoelectric quartz crystal disk coated with a gold electrode (100 nm thick) on the underside and an active surface layer of gold.

QCM-D tubing was decontaminated by flowing a solution of 2 vol \% Hellmanex solution (Hellma GmbH \& Co.) for 30 minutes, then $\mathrm{mQ}$ water for 30 minutes and finally air for 5 minutes.

All QCM-D crystals were optically polished with a root-mean-square roughness less than $3 \mathrm{~nm}$. Crystals were decontaminated by UV-ozonation for $10 \mathrm{~min}$, treated with 1:1:5 (vol) solution of ammonium hydroxide, hydrogen peroxide and water at $75^{\circ} \mathrm{C}$ for 5 minutes, submerged in water and rinsed with ethanol.

To immobilize the protein, clean crystals were placed in a $10 \mathrm{mM}$ solution of mercaptoundecanoic acid in methanol overnight, rinsed with methanol and dried.

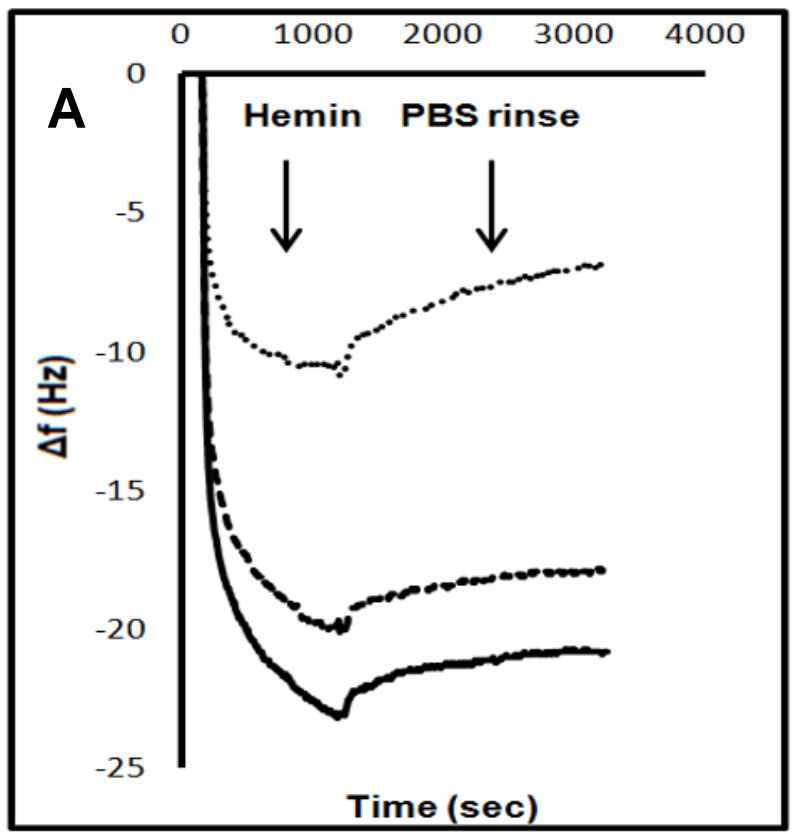

Crystals were then submerged in a solution of $5 \mathrm{mM} 1$ Ethyl-3-(3-dimethylaminopropyl)carbodiimide/ $N$ -

Hydroxysuccinimide (EDC/NHS) in $\mathrm{mQ}$ water for 2 hours at $4^{\circ} \mathrm{C}$, rinsed with PBS and placed in the solution containing glycated protein sample or the non-glycated control for 2 hours at $4^{\circ} \mathrm{C}$. Then, the crystals were rinsed with $\mathrm{mQ}$ water and placed in a solution of $50 \mathrm{mM}$ ethanolamine for 2 hours at $4^{\circ} \mathrm{C}$ [10]. Crystals were rinsed with $\mathrm{mQ}$ water and dried with $\mathrm{N}_{2}$ gas and then mounted in a liquid flow cell $(40 \mu \mathrm{L})$ and operated at $4.95 \mathrm{MHz}$.

A stable baseline was obtained by flushing the QCMD flow cell with PBS for 1 hour. Once this baseline was obtained, hemin $(30 \mu \mathrm{M})$ in PBS was passed through the flow cells, followed by a PBS rinse. After a stable baseline was achieved, the surface was re-exposed to the hemin solution, followed by a second PBS rinse. Flow cell temperature was fixed at $25.00 \pm 0.02{ }^{\circ} \mathrm{C}$, and a peristaltic pump (Ismatec ISM935C) was used to flow solution through the cell at a constant rate of $300 \mu \mathrm{L} / \mathrm{min}$. Details of QCM-D principles can be found elsewhere [11].

\section{Results and discussion}

In all cases, when hemin was exposed to the HSA functionalized surface, real-time QCM-D data showed a decrease in frequency and an increase in dissipation. This indicated an increase in mass on the sensor surface as result of hemin deposition. Following the first exposure to the hemin solution, the sensor surface was rinsed with PBS, but frequency values did not return to zero, despite the fact that the HSA-hemin interaction should be entirely reversible with a dissociation constant of approximately $10 \mathrm{nM}$ (figure 1A) [9]. This suggested a considerable

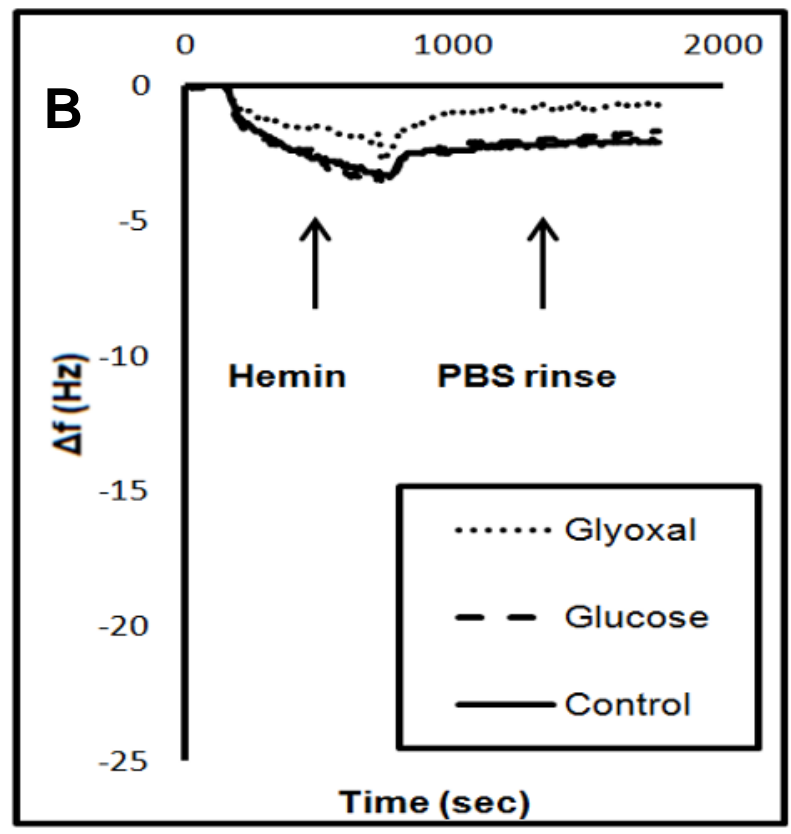

Fig 1. A. Quartz crystal microbalance data showing changes in frequency over time as hemin deposits onto HSA functionalized surfaces that have been pre-glycated with glyoxal or glucose for 19 days. The control was incubaed for 19 days under the same conditions in the absence of sugars. The surface has not been pre-exposed to hemin. B. The same surfaces from A, now capped with hemin, were exposed to hemin for a second time, followed by a PBS rinse. 
amount of irreversibly bound mass on the surface, which we attribute to an interaction between the carboxylic ends of the hemin molecule and two alcohols on the surface, originating from ethanolamine used during protein immobilization [12].

During the second exposure of hemin to the surface, reduced unintended irreversible interactions were observed. The first exposure of the surface to hemin effectively blocked the intrusive alcohol groups on the surface. With these alcohol groups blocked, the mass contribution of unintended irreversible binding was essentially eliminated during the second exposure of the surface to hemin (figure 1B). HSA incubated with glyoxal had a diminished hemin binding capacity, suggesting that modification of HSA by dicarbonyls in diabetics may result in increased concentrations of free hemin available to bacteria. Because diabetics have higher levels of glyoxal in their blood, more glyoxylation is expected to occur in diabetics. Based on the information presented herein, this could result in increased iron availability for bacteria in diabetics when compared to nondiabetics and may contribute to elevated diabetic infection rates. When hemin was exposed to the surface for a second time, no differences between the samples glycated with glucose and the control were observed, which is consistent with other studies that suggest glucose does not impact HSA's affinity for hemin [8].

Eliminating some of the irreversible interactions alone was not enough to determine a $\mathrm{K}_{\mathrm{d}}$ consistent with literature values for the binding event occurring on the surface. This may be because HSA has additional, secondary binding sites for hemin that confound our measurements. Dockal et al. suggest that HSA has three total hemin binding sites [13].

As far as we know, existing methods do not take into account the potentially confounding effects of unintended binding events that are always present to some degree in surface measurements. We are currently developing an accurate model of binding interactions on QCM-D that account for changes in frequency due to unintended interactions. The ability to decouple unintended from intended interactions would establish the feasibility of using a QCM-D to accurately determine the binding affinity of protein-ligand interactions.

\section{Conclusion}

HSA was incubated for two weeks with glucose, glyoxal and in the absence of sugar. When hemin was applied to an HSA functionalized surface, significant irreversible changes in frequency were observed. However, if the surface was pre-exposed to hemin, the unintended irreversible interactions were minimized. We believe the first exposure of hemin capped unintended irreversible binding sites so that the change in frequency reported by
QCM-D measurements taken during the second exposure of hemin was largely caused by binding to reversible sites. Doing this allowed us to obtain more accurate data on the binding behavior of hemin to the surface. No differences were observed between the nonglycated control and HSA glycated with glucose, which primarily attaches to lysine residues. HSA glycated with glyoxal showed diminished hemin binding capacity. This suggests that modification of HSA by dicarbonyls, which primarily attach to arginine residues, in diabetics may result in increased concentrations of free hemin available to bacteria. This data supports our hypothesis that the implications of glycation can differ for sugars with varied structures.

\section{Acknowledgements}

This research was supported by the Pomona College Summer Undergraduate Research Program and a Hirsch Research Initiation Grant.

\section{References}

1. J. M. Fernandez-Real, J. M. Moreno, B. Chico, A. Lopez-Bermejo W Ricart, Diabetes Care. 30, 616621 (2007)

2. M. Haap, A. Fritsche, H. J. Mensing, H. U. Haring, M. Stumvoll, Ann. Intern. Med. 139, 869-871 (2003)

3. S. N. Rajpathak, J. P. Crandall, J. Wylie-Rosett, G. C. Kabat, T.E. Rohan, F.B. Hu, Biochim Biophys Acta. 1790, 671-681 (2009)

4. J. K. Shetty, M. Prakash, M., M.S. Ibrahim, Ind. J. Clin. Biochem. 23, 67-70 (2008)

5. P.J. Thornalley, A. Langborg, H.S. Minhas, Biochem. J. 344, 109-116 (1999)

6. N. Iberg, R. Fluckinger, J. Biol. Chem. 261, 1354213545 (1986)

7. N. Ahmed, P. J. Thornalley, Ann N Y Acad Sci. 1043, 260-266 (2005)

8. N. Shaklai, R.L. Garlick, H.F. Bunn, J. Biol. Chem. 259, 3812-3817 (1984)

9. P.A. Zunszain, J. Ghuman, T. Komatsu, E. Tsuchida, S. Curry, BMC Struct. Biol. 3 (2003)

10. N. Patel, M.C. Davies, M. Hartshorne, R.J. Heaton, C.J. Roberts, S.J.B. Tendler, P.M. Williams, Langmuir. 13, 6485-6490 (1997)

11. M. Rodahl, F. Hook, A. Krozer, P. Brzezinski, B. Kasemo, Rev. Sci. Instrum. 66, 3924- 3930 (1995)

12. A.C. Maehly, Å. Åkeson, Acta Chem. Scand. 12, 1259 (1958)

13. M. Dockal, D.C. Carter, F. Rüker, J. Biol. Chem. 274 (1999) 